\title{
A copa em tweets: análise comparativa do uso das hashtags \#EstadaonaCopa e \#BairristanaCopa
}

Gabriela da Silva Zago ${ }^{1}$

1 Doutora em Comunicação e Informação pela Universidade Federal do Rio Grande do Sul (UFRGS). 


\section{Resumo}

Palavras-chave

Neste trabalho, procuramos analisar e comparar a utilização de hashtags de cobertura no Twitter por um site jornalístico e outro humorístico durante a Copa do Mundo de 2014: o jornal O Estado de S. Paulo, que utilizou a hashtag \#EstadaonaCopa para sua cobertura em sites de rede social, e o site satírico O Bairrista, que adotou a hashtag \#BairristanaCopa. O estudo foi operacionalizado a partir da combinação de observação com análise de redes sociais como métodos para compreender a estrutura e a dinâmica das redes em torno das respectivas hashtags. Resultados apontam para estruturas de rede semelhantes, porém com dinâmicas diferentes observadas em cada um dos casos estudados.

Jornalismo, humor, Twitter, redes sociais.

\section{Abstract}

In this paper, we aim to analyze and compare the use of hashtags for coverage on Twitter by a journalistic and a satirical website during the 2014 FIFA World Cup: the newspaper O Estado de S. Paulo, which used the hashtag \#EstadaonaCopa for the coverage on social network sites, and the satirical site O Bairrista, which adopted the hashtag \#BairristanaCopa. The study was conducted by combining observation and social network analysis as methods, in order to understand the network structure and dynamics of their respective hashtags. Results show similar network structures, but different dynamics observed in each of the cases studied.

\section{Keywords}

Journalism, humor, Twitter, social networks. 


\section{Introdução}

Durante a Copa do Mundo de 2014, inúmeros sites jornalísticos buscaram promover coberturas específicas sobre a temática, contando com seções para notícias relacionadas ao evento esportivo. Outras estratégias foram empregadas, em especial nos sites de rede social, como a criação de perfis específicos e a utilização de uma hashtag própria para a cobertura da Copa. Em sentido similar, outros sites de informação e de entretenimento buscaram criar espaços específicos para informações sobre o evento, com a criação de especiais, perfis e hashtags.

Neste trabalho procura-se analisar e comparar a utilização de hashtags de cobertura no Twitter por um perfil jornalístico e outro humorístico: o jornal $O$ Estado de S. Paulo, que utilizou a hashtag \#EstadaonaCopa para sua cobertura em sites de rede social, e o site satírico O Bairrista, que adotou a hashtag \#BairristanaCopa.

Para a operacionalização do estudo, emprega-se uma combinação de observação com análise de redes sociais, de modo a buscar compreender tanto a estrutura da rede de tweets em torno das hashtags, como também dinâmicas associadas à propagação de informações. Embora a hashtag tenha sido empregada por veículos específicos, outras pessoas poderiam retuitar informações ou usar a hashtag para outras finalidades.

O artigo está organizado da seguinte forma: no primeiro momento, discutese a utilização do Twitter para a circulação de informações. Na sequência, o foco recai sobre a análise de redes sociais enquanto método para compreender estruturas de redes. Após, apresentam-se os procedimentos metodológicos e os resultados obtidos com o estudo desenvolvido e, por último, as considerações finais.

\section{Circulação de informações no Twitter}

A circulação de informações pode ser observada em espaços públicos mediados como os sites de rede social. Para Ellison e Boyd (2013), redes sociais 
são espaços da web que permitem a articulação de conexões através de perfis de identificação única que interagem através de fluxos de conteúdos fornecidos por suas conexões no site. Em sites de redes sociais como o Twitter, a circulação de informações ocorre em um contexto de mídia espalhável (JENKINS; FORD; GREEN, 2013), em que a mídia tradicional não mais detém o monopólio de fazer circular conteúdos - outros atores podem contribuir, de diferentes formas, para a circulação. Os próprios veículos passam a investir em ferramentas para interação e participação do público, como no caso de utilizar redes sociais para postar chamadas para seus conteúdos, que podem ser compartilhadas e comentadas pelos usuários das redes.

As estratégias dos veículos jornalísticos também podem ser apropriadas por outros veículos informativos. No caso específico de um site que realiza a sátira de notícias - como é o caso de um dos sites investigados neste trabalho -, tal apropriação se torna ainda mais evidente na medida em que esses espaços utilizam o ethos jornalístico e elementos das notícias reais para produzir humor. Assim, faz sentido adotarem estratégias comunicativas típicas dos veículos jornalísticos.

Especificamente com relação ao Twitter, estudos anteriores (RECUERO; ZAGO, 2009) observaram que a ferramenta pode ser apropriada principalmente para duas finalidades distintas por seus usuários: para conversação e para informação. Em cada caso, o tipo de rede social resultante da apropriação será diferente. A rede de filiação é formada a partir dos seguidos e seguidores de cada usuário, que provêm acesso a determinadas informações, ainda que não haja interação efetiva entre os atores. As conexões são mantidas pelo sistema. Já a rede emergente é constituída pelas trocas comunicativas entre os atores registradas nas redes e representa situações em que de fato há interação entre as pessoas. As conexões são, assim, emergentes. Embora sejam redes diferentes em estrutura e dinâmica, ambas possuem valor para o usuário e permitem que tenham acesso a determinados recursos (Ibid.).

Para entender a estrutura e a dinâmica das redes associadas à circulação de informações no Twitter, um dos métodos que podem ser empregados é a Análise de Redes Sociais. 


\section{A abordagem metodológica da Análise de Redes Sociais}

A Análise de Redes Sociais (ARS) compreende um conjunto de métricas e técnicas de pesquisa utilizadas para descrever a relação entre os atores e suas conexões (SCOTT, 2013). No contexto dos sites de rede social, os atores são representados por perfis, e as conexões são estabelecidas a partir de relações de amizade típicas de cada ferramenta (no Facebook, é preciso adicionar alguém como "amigo"; no Twitter, é possível seguir um usuário sem que necessariamente o outro usuário siga de volta). O foco da ARS, assim, está em como se dá a relação entre os nós (WASSERMAN; FAUST, 1994).

Fragoso, Recuero e Amaral (2011) apontam como primeiro passo da análise em redes sociais "pensar quem serão considerados os atores e suas conexões, ou seja, o que será considerado uma conexão e o que será considerado um ator (ou um nó, que também poderia ser uma instituição) e em qual medida" ( $p$. 118). Neste trabalho, serão considerados como atores os indivíduos que fizeram tweets ou foram mencionados em tweets contendo as hashtags estudadas. Já as conexões serão consideradas a partir das relações de menção e retweet estabelecidas pelos tweets. Assim, se um tweet de um ator A faz um retweet de um ator B, pode-se dizer que há uma conexão entre eles, e tanto ator A quanto B passam a integrar a rede como nós.

Ainda que a ARS disponha de diversas métricas e técnicas para compreender a rede como um todo e as propriedades de cada um de seus nós, neste trabalho focaremos principalmente nas métricas apresentadas no Quadro 1. 


\begin{tabular}{|c|c|c|}
\hline \multirow{3}{*}{ Rede } & Densidade & $\begin{array}{l}\text { A densidade é uma métrica de rede que } \\
\text { descreve o quanto os nós de uma rede es- } \\
\text { tão interconectados. Quanto maior a den- } \\
\text { sidade, mais conectados estão entre si. }\end{array}$ \\
\hline & $\begin{array}{l}\text { Distância } \\
\text { geodésica }\end{array}$ & $\begin{array}{l}\text { A distância geodésica se refere ao diâme- } \\
\text { tro da rede e à distância entre os nós. A } \\
\text { distância representa o caminho entre dois } \\
\text { nós; já a geodésica representa o menor } \\
\text { caminho entre um par de nós (FREEMAN, } \\
\text { 1979). }\end{array}$ \\
\hline & Modularidade & $\begin{array}{l}\text { A modularidade é uma métrica que permi- } \\
\text { te identificar grupos de nós de acordo com } \\
\text { a força das conexões entre os atores. Nós } \\
\text { incluídos em um mesmo módulo possuem } \\
\text { conexão mais forte entre si do que com } \\
\text { outros atores da rede. }\end{array}$ \\
\hline Nó & Grau de conexão & $\begin{array}{l}\text { O grau de conexão representa a força da } \\
\text { conexão entre os nós. Quanto mais conec- } \\
\text { tado a outros nós, maior o grau de cone- } \\
\text { xão de um determinado ator. O grau de co- } \\
\text { nexão pode ser desdobrado em conexões } \\
\text { recebidas (indegree) e conexões enviadas } \\
\text { (outdegree). }\end{array}$ \\
\hline
\end{tabular}

Quadro 1: Métricas de rede consideradas no trabalho

Assim, os procedimentos da ARS permitem compreender a relação entre usuários que postaram sobre um determinado assunto - mais especificamente, se há menção a outros usuários, e em torno de quais usuários a rede está centrada. A ARS também pode ser utilizada para compreender a estrutura da circulação de informações jornalísticas (ZAGO, 2014). A circulação pode ser estudada a partir de relações entre os conteúdos postados, ou entre indivíduos que colocaram em circulação postagens abordando desdobramento de notícias. Tal abordagem permite identificar nós centrais na circulação de determinados acontecimentos jornalísticos, bem como o quanto a rede está centrada em determinados nós.

Além de permitir o mapeamento da estrutura, a ARS pode ser combinada com outros métodos para se buscar compreender dinâmicas associadas à circulação de informações. Ao mesmo tempo em que a estrutura permite apreender propriedades de uma rede em um determinado período, o estudo 
das dinâmicas permite perceber mudanças que ocorrem em uma rede ao longo do tempo. Nesse sentido, o conceito de dinâmica está relacionado aos comportamentos coletivos que podem ser observados numa determinada rede social (RECUERO, 2006), e abrange, entre outros aspectos, os processos de cooperação, conflito e competição entre os atores (PRIMO, 2005).

Cooperação e conflito são duas dinâmicas complementares que podem ser observadas nas redes (Idem). Enquanto na cooperação a ação de vários usuários é concentrada em torno de um esforço comum, o conflito ocorre quando não há consenso entre os atores envolvidos. Já a competição guarda relação com uma potencial disputa entre atores na rede. A competição é, muitas vezes, uma disputa por visibilidade (RECUERO, 2006).

A seguir apresentam-se os procedimentos metodológicos empregados para compreender a estrutura e as dinâmicas associadas à utilização de hashtags para cobertura da Copa pelos sites Estadão e O Bairrista.

\section{Procedimentos metodológicos}

Durante a Copa do Mundo de 2014, dados associados à circulação de informações sobre o evento foram coletados diariamente. Um total de 272 conjuntos de dados foram obtidos a partir de hashtags e palavras-chave usadas para descrever o Mundial (ZAGO, 2014). Desse universo, escolheu-se observar mais atentamente as redes em torno de hashtags de cobertura propostas por veículos. Devido às diferenças nas naturezas dos veículos e nas apropriações observadas, as hashtags escolhidas foram \#EstadaonaCopa, usada pelo jornal O Estado de S. Paulo, e \#BairristanaCopa, usada pelo site satírico O Bairrista.

Os dados coletados para a Análise de Redes Sociais foram obtidos através da ferramenta NodeXL (NODEXL, 2015), um plugin para Excel que pode ser utilizado para coletar e analisar dados provenientes de sites de rede social (SMITH et al., 2010). A análise foi feita usando o próprio NodeXL e, posteriormente, a visualização da rede foi gerada usando o Gephi (2015), um software open source voltado para a análise e a visualização de redes. 
O NodeXL coleta dados no Twitter a partir de uma hashtag ou palavra-chave utilizando a API de busca do Twitter. Os tweets coletados são de um período anterior ao momento da consulta. Em ambos os casos, as coletas foram realizadas no dia 14 de junho de 2014 e abrangem um período de cerca de 24 horas.

\section{Resultados e discussão}

A seguir são apresentados os resultados obtidos pela análise da circulação de cada uma das hashtags isoladamente, para, na sequência, realizar a comparação entre as duas formas de cobertura.

\section{Hashtag para cobertura de veículo jornalístico: \#EstadaonaCopa}

O primeiro recorte utilizado no presente trabalho corresponde ao emprego da hashtag \#EstadaonaCopa pelo veículo Estadão anterior, concomitante e posteriormente à Copa do Mundo de 2014 para se referir à cobertura do evento esportivo. A hashtag foi usada pelo jornal em diversos sites de rede social, como Twitter, Facebook e Instagram.

Por ser usada pelo veículo, a hashtag também foi utilizada por usuários com os quais interagia, seja por meio de retweets ou de menções. O próprio veículo promoveu iniciativas de participação do público com o uso da hashtag, como no caso de pedir que postassem fotos de seus álbuns da Copa no Instagram com a hashtag \#EstadaonaCopa (LEITORES..., 2014).

Outros veículos também adotaram estratégias similares de cobertura jornalística durante a Copa, com hashtags como \#FolhanaCopa, do jornal Folha de São Paulo, \#GauchanaCopa, da rádio Gaúcha, e \#NaCopaZH, do jornal Zero Hora.

A coleta de tweets contendo a hashtag \#EstadaonaCopa² foi realizada no dia 14 de junho de 2014 usando a ferramenta NodeXL. A coleta foi realizada às 09h51min e compreende um período de quase 24 horas, com tweets a partir das $12 \mathrm{~h} 24$ do dia 13 de junho. Ao todo foram obtidas 2.005 conexões (relações de 
menção ou retweet) e 1.338 nós (autores dos tweets e usuários mencionados ou retuitados) a partir de tweets contendo a hashtag \#EstadaonaCopa. Dessas 2.005 conexões, 1.475 são únicas (ou seja, alguns usuários postaram mais de um tweet estabelecendo a mesma conexão dentro da amostra, da mesma forma que alguns tweets estabeleceram mais de uma conexão ao mencionar 2 ou mais atores).

No conjunto de dados referentes à hashtag \#EstadaonaCopa, ao todo foram identificados 24 componentes (grupos de nós conectados entre si), sendo que 15 possuem uma única conexão. O componente com maior número de nós possui 1.299 nós interligados entre si por relações de menção ou retweet. Por se tratar de uma rede em torno de uma hashtag específica, o número de componentes é bastante reduzido. A maior parte dos nós (1.299 de um total de 1.338 nós, o que corresponde a $97 \%$ o total) está incluída em um único componente ao redor do perfil principal no Twitter do jornal @estadao.

A distância geodésica máxima entre os nós é de 4 (diâmetro da rede), e a distância geodésica média é de 2,052618. A rede é pequena em termos de distância entre os nós, na medida em que são necessários no máximo 4 "pulos" para percorrer a rede toda. A densidade do grafo é de 0,000897755 (o número vai de 0 a 1 ). No recorte, o maior indegree é 1.255 (número de menções recebidas), e o maior outdegree é 7 (número de tweets feitos).

As hashtags mais presentes no conjunto de dados são, obviamente, \#EstadãonaCopa, em 1.182 tweets, e \#EstadaonaCopa, em 513 tweets. Esse resultado era esperado tendo em vista se tratar de uma rede centrada em torno de uma hashtag específica. Outras hashtags aparecem em menor quantidade, todas associadas a times de futebol, como \#NED com 448 menções, \#ESP, com 447, \#CHI, com 107 e \#AUS, também com 107 menções, o que traz indícios de que o veículo utilizou as hashtags oficiais das seleções no Twitter durante a sua cobertura da Copa.

Dentre os usuários com maior indegree no recorte (Quadro 2), além do predomínio óbvio de @estadao, outros atores aparecem, como @dilmabr, @ 
neymarjr e @cristiano, personagens citados nas manchetes do jornal publicadas no microblog, ou @necromorty, @fitzca e @meiaseis, usuários que fizeram retweets ao jornal contendo a hashtag.

\begin{tabular}{|c|c|}
\hline Usuário & Número de menções \\
\hline @estadao & 1255 \\
\hline @dilmabr & 127 \\
\hline @necromorty & 40 \\
\hline @fifaworldcup & 22 \\
\hline @neymarjr & 19 \\
\hline @cristiano & 18 \\
\hline @fitzca & 18 \\
\hline @estadaoesporte & 11 \\
\hline @meiaseis & 10 \\
\hline
\end{tabular}

Quadro 2: Nós mais centrais no recorte (maior grau de conexão indegree)

Um exemplo de retweet ao usuário @necromorty contendo a hashtag \#EstadãonaCopa pode ser visto abaixo:

RT @Necromorty: ¿\#MundialBrasil2014? (VÍA @Estadao) Artefato explode próximo à Câmara de Vereadores \#EstadãonaCopa \#VzlaViveEIFutbol

Já no exemplo a seguir podemos ver um retweet de atualização do jornal com menções aos atores envolvidos na notícia (os jogadores @neymarjr e @cristiano):

RT @Estadao: .@neymarjr supera média de gols de Messi e @Cristiano Ronaldo http://t.co/WE6MgChWlr \#EstadãonaCopa

No caso acima, o tweet original do jornal se inicia com o caractere ponto (.). Essa medida demonstra o domínio por parte do jornal da linguagem do Twitter, uma vez que, ao iniciar um tweet com "@", ele somente será exibido para as pessoas que também seguem o usuário.

A visualização da rede (Figura 1) foi gerada no Gephi usando o layout Force Atlas. Os nós estão coloridos por modularidade. O tamanho do nó representa 
o indegree - quando maior o nó, mais conexões (menções ou retweets) o usuário recebeu. Os pontos isolados ao redor do grafo representam usuários que postaram tweets sem mencionar outros usuários.

Figura 1: Grafo da rede de tweets, retweets e menções com a hashtag \#EstadaonaCopa nos dias 13 e 14 de junho de 2014

O grafo possui uma estrutura bastante peculiar, uma vez que está centrado em um único usuário (@estadao, em azul, no centro). Há pouquíssimos nós desconectados (pontos isolados nas bordas do grafo). Possui uma estrutura com núcleo central contendo usuários que mencionaram o jornal @estadao mais de uma vez no período (dois ou mais retweets). Já os usuários que mencionaram o veículo apenas uma vez aparecem na parte externa do hub. O pequeno grupo de 
nós acima, à esquerda, na parte externa do grafo (estrutura parecida com uma aranha), corresponde a retweets ao usuário @meiaseis, que usou a hashtag, porém não mencionou o perfil do jornal Estadão em seu tweet. Dentro do grafo principal, é possível identificar tweets com menção a @dilmabr, em verde, @ necromorty em vermelho, e @fifaworldcup, em amarelo.

\section{Hashtag para cobertura de site humorístico: \#BairristanaCopa}

O segundo recorte utilizado neste trabalho corresponde ao emprego da hashtag \#BairristanaCopa pelo site satírico O Bairrista (O BAIRRISTA, 2015). O veículo satírico é conhecido no Rio Grande do Sul por satirizar o jornal Zero Hora, apresentando notícias humorísticas falsas que se passam no estado, representado como se fosse um país autônomo em relação ao "Brazil" (grafado com " $z$ " pelo site, usando o tratamento de um território internacional).

A coleta de tweets contendo a hashtag \#BairristanaCopa foi realizada no dia 14 de junho de 2014 usando a ferramenta NodeXL. A coleta foi realizada às 16 h37 e compreende um período de 24 horas, a partir das $16 \mathrm{~h} 01$ do dia 13 de junho. Ao todo, foram obtidas 1552 conexões (relações de menção ou retweet) e 1014 nós (autores dos tweets e usuários mencionados ou retuitados), a partir de tweets contendo a hashtag \#BairristanaCopa. Das 1552 conexões, 983 são únicas.

No conjunto de dados referentes à hashtag \#BairristanaCopa, ao todo foram identificados 17 componentes, sendo que 14 possuem uma única conexão. O componente com maior número de nós possui 996 nós interligados entre si por relações de menção ou retweet. Por se tratar de uma rede em torno de uma hashtag específica, o número de componentes é bastante reduzido. A maior parte dos nós (996 de um total de 1014) está interconectada entre si em torno do perfil principal no Twitter do site @O_Bairrista.

A distância geodésica máxima entre os nós é de 6 (diâmetro da rede), e a distância geodésica média é de 2,777267. Essa rede é "maior" em termos de distância entre os nós do que a rede em torno da hashtag \#EstadaonaCopa, na medida em que são necessários até 6 "pulos" para percorrer a rede toda. Mesmo 
assim, a densidade do grafo é de 0,00109815, maior que a observada na rede anterior. No recorte, o maior indegree é 633 (número de menções recebidas), e o maior outdegree é 5 (número de tweets feitos).

As hashtags mais presentes no conjunto de dados são, obviamente, \#BairristanaCopa, em 1.453 tweets, e \#bairristanacopa, em 41 tweets. Tal resultado era esperado tendo em vista se tratar de uma rede centrada em torno de uma hashtag específica. Outras hashtags aparecem em menor quantidade, como \#URU, em 171 menções, \#WorldCup2014, em 14 menções, e \#BamoURU, em 4 tweets.

Dentre os usuários com maior indegree no recorte (Quadro 3), além do predomínio óbvio de @O_Bairrista, outros atores aparecem, com destaque para @juarezroth e @sandrosotigol. Os dois perfis fazem parte da equipe do site O Bairrista. São descritos como perfis falsos dos futebolistas Sandro Sotili (atacante aposentado gaúcho que atuou em diversos times do estado) e Celso Roth (treinador gaúcho que já comandou, entre outras equipes, o Internacional e o Grêmio. Seu nome do meio é Juarez).

\begin{tabular}{|c|c|}
\hline Usuário & Número de menções \\
\hline @O_Bairrista & 633 \\
\hline @juarezroth & 250 \\
\hline @sandrosotigol & 214 \\
\hline @pazinatto89 & 13 \\
\hline @edubisotto & 9 \\
\hline
\end{tabular}

Quadro 3: Nós mais centrais no recorte (maior grau de conexão indegree)

Um retweet ao perfil @juarezroth pode ser visto no exemplo abaixo. Ainda que não haja menção ao perfil principal do site, o retweet inclui a hashtag \# BairristanaCopa.

RT @JuarezRoth: É muito gol nessa Copa. Cadê os 0x0??? \#BairristanaCopa 
Atlas. Os nós estão coloridos por modularidade. O tamanho do nó representa o indegree - quando maior o nó, mais conexões (menções ou retweets) o usuário recebeu. Os pontos isolados ao redor do grafo representam usuários que postaram tweets sem mencionar outros usuários.

Figura 2: Grafo da rede de tweets, retweets e menções com a hashtag \#BairristanaCopa nos dias 13 e 14 de junho de 2014

O grafo possui uma estrutura ligeiramente diferente da observada na rede em torno da hashtag \#EstadaonaCopa, pois, em vez de estar centrada em um único perfil, aparece com três grandes grupos de nós: o grupo verde, no centro, em torno do perfil @O_Bairrista, o grupo azul, na parte inferior, centrado no perfil @sandrosotigol, e o grupo vermelho, na parte superior, em torno do perfil @juarezroth. Ainda que os tweets estejam distribuídos, os três perfis possuem 
relação direta com o site satírico $O$ Bairrista. Ao redor do grafo, há os 14 nós isolados que usaram a hashtag em seus tweets, sem, contudo, mencionar ou ser mencionado por nenhum outro usuário presente no recorte.

\section{Discussão}

A estrutura das duas redes possui alguns elementos em comum, como o fato de contarem com poucos componentes (há um núcleo central com menções ao perfil principal dos sites no Twitter - @estadao e @O_Bairrista), porém dinâmicas diferentes podem ser observadas, com o predomínio da utilização para participação do público na hashtag \#BairristanaCopa e de retweet de últimas notícias na hashtag \#EstadaonaCopa.

Enquanto na rede em torno da hashtag \#EstadaonaCopa é possível observar a maioria dos tweets contendo links para o jornal O Estado de S. Paulo (apenas 107 tweets não apresentam links), na rede em torno do \#bairristanacopa há apenas 2 tweets com links. Além disso, a rede \#bairristanacopa apresenta 45 relações de replies (resposta a outro usuário), ao passo que a rede \#EstadaonaCopa possui apenas 3 replies. A quase ausência de links e a presença expressiva de respostas a outros usuários são indícios de que a rede do \#BairristanaCopa envolveu mais participação do que compartilhamento de informações (ao menos dentro do recorte observado).

Uma das formas mais comuns de participação foi através da sugestão de placares para jogos futuros, como no caso da partida Uruguai X Costa Rica, que estava sendo realizada às $16 \mathrm{~h}$ do dia 14 de junho no momento da coleta dos dados. No contexto do perfil de humor do Bairrista, a aposta era que o Uruguai, país bastante próximo ao Rio Grande do Sul, vencesse a partida, o que explica também a presença de hashtags como \#URU e \#BamoURU no conjunto de dados analisados.

A participação foi estimulada a partir de um tweet feito pelo perfil falso @ sandrosotigol pouco antes do início da partida do Uruguai: 
QUAL É O TEU PALPITE PARA O JOGO DA SELECCIÓN? QUEM ACERTAR EU DOU RT (TEM QUE TER A TAG \#BairristanaCopa @O_Bairrista)

Ao participar, os usuários mencionavam muitas vezes ambos os perfis (@ sandrosotigol e @O_Bairrista), o que contribuiu para a configuração da estrutura da rede em torno de três perfis, bem como para reduzir a densidade total da rede.

@SandroSotigol @O_Bairrista 3 x 0 \#URU \#BairristanaCopa

Assim, é possível observar que os dois sites utilizaram hashtags de cobertura no Twitter durante a Copa com propósitos e apropriações diversas. De um lado, temos um veículo jornalístico tradicional que utiliza a hashtag para compartilhar links para notícias (com a apropriação mais típica sendo para retweet de notícias). De outro, temos um site satírico que utiliza a hashtag para produzir humor sobre a Copa e estimular a participação do público.

Em termos de tipos de redes observadas, seria possível inferir que a rede em torno da hashtag \#EstadaonaCopa assume contornos de uma rede de filiação, com caráter informativo - mesmo que não haja tanta conversação no recorte, os retweets a mensagens com links provêm e conferem acesso às notícias. Já na rede em torno da hashtag \#BairristanaCopa pode-se perceber a formação de uma rede emergente, com caráter conversacional. O foco, aqui, parece ser mais no sentido de participar e difundir o humor do que propriamente dar ou ter acesso a informações.

\section{Considerações finais}

O trabalho procurou analisar e comparar a utilização de hashtags de cobertura no Twitter por um site jornalístico e outro humorístico durante a Copa do Mundo de 2014. As hashtags escolhidas foram \#EstadaonaCopa, utilizada pelo jornal $O$ Estado de S. Paulo, e \#BairristanaCopa, adotada pelo site satírico O Bairrista.

Através da observação da estrutura e das dinâmicas associadas ao emprego de cada hashtag, foi possível identificar semelhanças e diferenças nas 
apropriações e nos propósitos de cada cobertura. Assim, enquanto na rede em torno da hashtag \#EstadaonaCopa predomina a circulação de informações por meio do retweet de notícias do portal Estadão com links, na rede em torno da hashtag \#BairristanaCopa predomina humor e participação do público.

As estruturas e dinâmicas observadas permitem traçar algumas considerações. Ainda que sites satíricos possam empregar estratégias de veículos jornalísticos tradicionais (como ao propor, também, uma hashtag específica para cobertura da Copa), as apropriações observadas, tanto por parte do site satírico quanto por parte do veículo jornalístico, podem ser diferentes. Tais diferenças podem se dar tanto na produção (o que cada perfil posta e quem faz as postagens) quanto na recepção, ao estimular a participação do público na cobertura realizada.

Além disso, observa-se que a ARS pode ser uma ferramenta útil para compreender redes em torno de determinadas hashtags, na medida em que permite identificar estruturas de rede a partir das relações entre os tweets analisados. No entanto, mesmo que se mapeie a estrutura da rede, não se descarta que a análise seja complementada por um estudo das dinâmicas observadas, ou seja, de como a rede se comporta ao longo do tempo e como os usuários interagem entre si. 


\section{Referências}

ELLISON, N. B.; BOYD, D. Sociality through social network sites. In: DUTTON, W. H. (Org.). The Oxford handbook of internet studies. Oxford: Oxford University Press, p.151-172, 2013.

FRAGoso, S.; ReCUeRO, R.; AMARAL, A. Métodos de pesquisa para internet. Porto Alegre: Sulina, 2011.

FREEMAN, L. Centrality in social networks: conceptual clarification. Social Networks, v. 1, p. 215-239, 1979.

GEPHI. Disponível em: <https://gephi.github.io/>. Acesso em: 28 out. 2015.

JENKINS, H.; FORD, S.; GREEN, J. Spreadable media. Nova Iorque: NYU Press, 2013.

LEITORES mostram sua coleção de figurinhas \#EstadaoNaCopa. Disponível em: <http://blogs.estadao.com.br/dentro-da-rede/2014/?doing_wp_cron=1406736 018.3082880973815917968750>. Acesso em: 30 jul. 2014.

NODEXL: Network Overview, Discovery and Exploration for Excel. Disponível em: <lhttp://nodexl.codeplex.com>. Acesso em: 28 out. 2015.

O BAIRRISTA. Disponível em: <http://www.obairrista.com>. Acesso em: 28 out. 2015.

PRIMO, A. Conflito e cooperação em interações mediadas por computador. Contemporânea, v. 3, n. 1, p. 38-74, 2005. Disponível em: <http://www.ufrgs. br/limc/PDFs/conflito.pdf>. Acesso em: 25 ago. 2014. 
RECUERO, R. Dinâmicas de redes sociais no Orkut e capital social. Razon y Palabra, n. 52, 2006. Disponível em: <http://www.razonypalabra.org.mx/ anteriores/n52/11daCunha.pdf>. Acesso em: 25 ago 2014.

RECUERO, R.; ZAGO, G. Em busca das "redes que importam": redes sociais e capital social no Twitter. Líbero, n. 12, v. 24, p. 81-94, 2009.

SCOTT, J. Social network analysis. 3. ed. Londres: Sage Publications, 2013.

SMITH, M. et al. NodeXL: a free and open network overview, discovery and exploration add-in for Excel 2007/2010, 2010. Disponível em: <http://nodexl. codeplex.com/>. Acesso em: 20 set. 2014.

WASSERMAN, S.; FAUST, K. Social network analysis: methods and applications. Cambridge: Cambridge University Press, 1994.

ZAGO, G. Circulação e recirculação de narrativas do acontecimento no jornalismo em rede: a Copa do Mundo de 2014 no Twitter. 2014. 217 f. Tese (Doutorado)Universidade Federal do Rio Grande do Sul, Porto Alegre, 2014.

submetido em: 17 abr. 2015 | aprovado em: 07 ago. 2015 